\title{
Effect of oligosaccharides on glucose consumption by Rhodobacter sphaeroides in polyhydroxyalkanoate production from enzymatically treated crude sago starch
}

\begin{abstract}
A method of producing a bacterial polyester, polyhydroxyalkanoate (PHA), from enzymatically treated crude sago starch using the photosynthetic bacterium Rhodobacter sphaeroides (IFO 12203) is described. When Ü-amylase and glucoamylase were used in sequence to saccharify sago starch, the isomaltose thus produced was found to enhance glucose consumption by the cells, resulting in increased volumetric PHA productivity. On the other hand, treatment with a mixture of glucoamylase and pullulanase during the saccharification of sago starch resulted in the production of glucose but no isomaltose. Using this solution, glucose consumption by R. sphaeroides was lower than that using the solution treated with Ŭ-amylase + glucoamylase. These results suggested that isomaltose (with 1-6 bonding) contributed to the enhancement of the glucose consumption rate and PHA yield. This was experimentally confirmed by cultivating R. sphaeroides in a medium containing glucose with either isomaltose or malto-oligosaccharides without 1-6 bonding.
\end{abstract}

Keyword: Amylases; Oligosaccharides; PHA; Photosynthetic bacteria; Polyhydroxyalkanoate; Rhodobocter sphaeroides; Sago; Starch 